\title{
ARTICLE
}

\section{Facing the Forensic Challenge of Cannabis Regulation: A Methodology for the Differentiation between Hemp and Marijuana Samples}

\section{Presumptive and confirmatory methods for hemp and marijuana analysis}

Virgínia Martins Carvalho1* $\mathbb{\text { D }}$, Fernando Gomes de Almeida ${ }^{1,2}$ iD, Andrey Fabiano Lourenço de Aguiar $^{1}$, Fernanda Cardoso dos Santos Pereira ${ }^{1}$, Ernesto Díaz Rocha ${ }^{1}$ iD

${ }^{1}$ Laboratório de Análises Toxicológicas, Departamento de Análises Clínicas e Toxicológicas, Faculdade de Farmácia, Universidade Federal do Rio de Janeiro, Av. Clementino Fraga Filho, 373, Ilha do Fundão, Cidade Universitária, 21941-902, Rio de Janeiro, RJ, Brazil

${ }^{2}$ Laboratório Geral de Perícias Químicas, Instituto de Criminalística Carlos Éboli, R. Pedro I, 28, 20060-050, Rio de Janeiro, RJ, Brazil

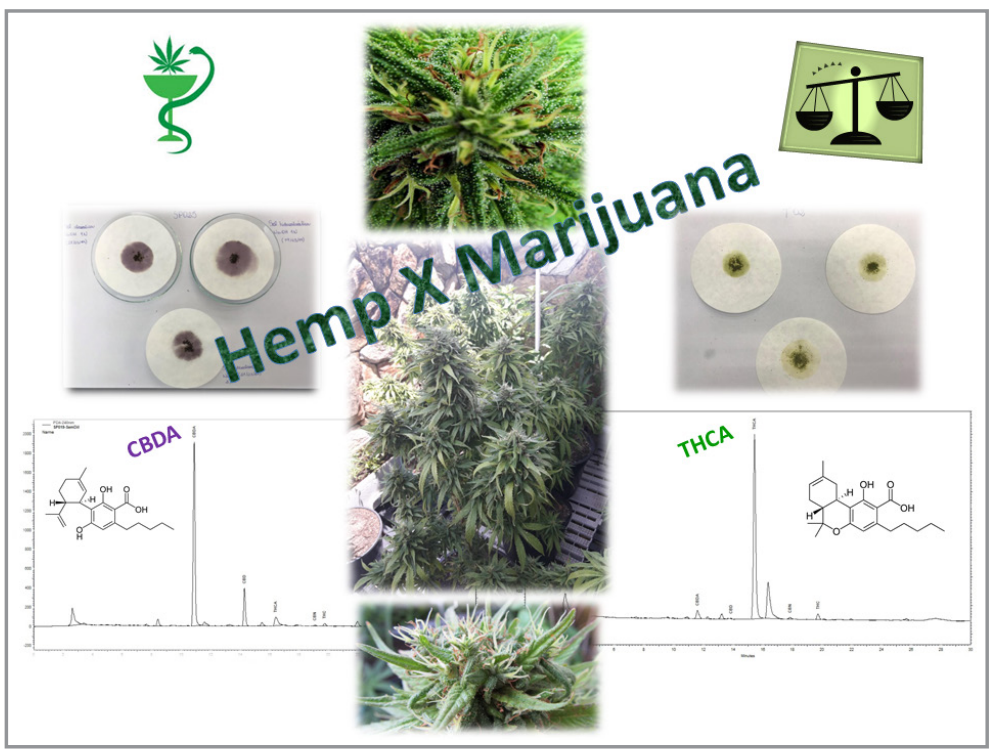

Access to medical and recreational cannabis has been regulated in several countries. Cannabinoids are secondary metabolites used as chemical markers of Cannabis genus plants. Tetrahydrocannabinol (THC) and cannabidiol (CBD) are the most abundant cannabinoids and their proportion is used to differentiate hemp $(\mathrm{THC} / \mathrm{CBD}<1)$ from marijuana (THC/CBD > 1) varieties. Brazilian sanitary regulations permit prescription, importation and sale of cannabis-derived products by pharmaceuticals distributors and drugstores, even though their crops are still prohibited, and the current scenario is characterized by marijuana trafficking, legal trade of medical cannabis products, and cultivation and production of cannabis-derived products by patients and non-governmental organizations (NGOs), with or without judicial authorizations. Medical cannabis regulation is in progress and there is an urgent need to implement analytical methods for monitoring the chemical profile of cannabis crops. Thus, the goal of the present study was to propose a methodology based on presumptive and confirmatory methods to differentiate the two principal chemovars of Cannabis genus plants, i.e., CBD-rich (hemp) and THC-rich (marijuana). A color test and a validated high-performance liquid chromatography (HPLC) method were applied to six cannabis samples cultivated by patients with judicial authorization. The methodology proved

Cite: Carvalho, V. M.; de Almeida, F. G.; de Aguiar, A. F. L.; Pereira, F. C. S.; Rocha, E. D. Facing the Forensic Challenge of Cannabis Regulation: A Methodology for the Differentiation between Hemp and Marijuana Samples - Presumptive and confirmatory methods for hemp and marijuana analysis. Braz. J. Anal. Chem., 2022, 9 (34), pp 162-176. doi: http://dx.doi. org/10.30744/brjac.2179-3425.AR-42-2021

Submitted 23 March 2021, Resubmitted 25 May 2021, Accepted 03 June 2021, Available online 23 June 2021. 
to be useful for forensic purposes and for quality control of medical cannabis. Plants cultivated by patients showed three profiles, classified as THC-rich, CBD-rich and approximately 1:1 THC/CBD ratio.

Keywords: cannabinoids, hemp, marijuana, medical cannabis, forensic methodology

\section{INTRODUCTION}

Cannabis access has been regulated in several countries and many of them have adopted two different framework regulations for the medical and recreational market [1,2]. Before the revolution in its regulation, cannabis fibers had already been legalized in United States of America, Canada and many European countries. Botanically, plants of the genus Cannabis are considered to be either monospecific with several subspecies, such as Cannabis sativa subsp. sativa, Cannabis sativa subsp. indica, Cannabis sativa subsp. ruderalis, Cannabis sativa subsp. spontanea, Cannabis sativa subsp. Kafiristanca [3], or a multispecies genus, such as Cannabis sativa and Cannabis indica [4,5]. Although the taxonomic organization is still debatable, all Cannabis genus plants contain cannabinoids (terpenephenolic compounds) as chemical markers. More than one hundred cannabinoids have been described, but the most abundant are tetrahydrocannabinolic acid (THCA) and cannabidiolic acid (CBDA), both derived from cannabigerolic acid (CBGA). CBGA is converted to THCA, CBDA and cannabicromenic acid (CBCA) by THCA, CBDA and CBCA synthases [6].

Acid forms can be converted to decarboxylated derivatives, such as the neutral cannabinoids THC, CBD and cannabinol (CBN), which is a by-product from heat-induced THC degradation and heat-combustion, or accumulated as a result of plant-aging. Although neutral forms are the most pharmacologically active due to their role as modulators of several components of the endocannabinoid system, acid forms have also been associated with therapeutic properties [7].

The proportion between THC and CBD content in plants is used to differentiate chemovars types. According to the United Nations Office on Drugs and Crimes (UNODC), plants are classified based on the concentration of their main phytocannabinoids. For forensic purposes, in a gas chromatographic analysis, if the peak area ratio of $[\mathrm{THC}+\mathrm{CBN}] /[\mathrm{CBD}]$ is $<1$, then the cannabis plant is considered to be a fibertype (hemp). If the ratio is $>1$, it is considered drug-type (marijuana) [3]. US and Canadian regulations only permit hemp cultivars containing less than $0.3 \%$ THC to be grown for textile, cosmetics and food supplement markets [1].

Unlike countries in North America and Europe, Brazil has never permitted the cultivation of cannabis fiber-type (hemp) crops. Considering that the Brazilian Federal Drug Law No. 11343/2006 does not define which compounds and plant chemotypes are illegal within its territory, the Brazilian Health Regulatory Agency on Sanitary Surveillance (Agência Nacional de Vigilância Sanitária-ANVISA) regulates cannabis and cannabinoid utilization by "Ordinance No. 344/98" and other specific sanitary bills. At the present, the use of medical cannabis is permitted only in the form of pharmaceutically formulated products and CBDrich extracts containing no more than $0.2 \%$ THC can be used for any pathological conditions. Cannabisderived products containing more than $0.2 \%$ THC are restricted to palliative care, when either other therapeutic alternatives fail to improve patients' welfare, or in the presence of irreversible or terminal clinical conditions.

Although cannabis crops are still prohibited, some patients and NGOs have been turning to the courts to obtain permission to cultivate cannabis plants and produce medicinal extracts. Thus, Habeas corpus (the mechanism of urgent protection against arbitrary detentions) has been granted as a preventive measure for protection of cannabis cultivation. Currently, dozens of patients are being granted Habeas corpus and some NGOs have obtained legal permission to cultivate, produce and supply cannabis extracts to hundreds of people with different disorders, with anecdotal claims that they are serving thousands of patients. In this unusual scenario, a project called Farmacannabis has been created at the Faculty of Pharmacy of the Federal University of Rio de Janeiro in order to ensure pharmaceutical support for patients who cultivate cannabis under Habeas corpus, and to monitor cannabis-based therapies for children with 
intractable epilepsy, patients with cancer, chronic pain, Alzheimer's, Parkinson's and other diseases [8]. Within the context of the Farmacannabis project, a methodology consisting of a presumptive color test and a confirmatory HPLC method has been developed, and the latter was validated, in order to differentiate CBD-rich and THC-rich cannabis samples.

Medical cannabis regulation requires improvement in forensic analytical methodologies. Thus, the present study proposes the use of this methodology as a tool to clarify cases related to drug trafficking, individual cultivation for medical purposes and sanitary crimes, such as the sale of products that do not obey sanitary specifications.

\section{MATERIALS AND METHODS \\ Chemicals and reagents}

All certified reference standards (CRS) for CBDA, CBD, THCA, THC and CBN were purchased from Cerilliant ${ }^{\circledR}$ (Texas, USA) and stored at $-20{ }^{\circ} \mathrm{C}$. Methanol, ultrapure water and n-hexane at HPLC grade were purchased from Scharlau Chemicals ${ }^{\circledR}$ (Barcelona, Spain) and ethanol absolute $(\geq 99.5 \%$, reagent grade) was obtained from Tedia Company ${ }^{\circledR}$ (Ohio, USA). Ammonium formate and sodium hydroxide, which were used for preparation of mobile phase and presumptive test, respectively, were both purchased from Loba Chemie Pvt. Ltd. (Mumbai, India).

\section{Sample collection}

Dried flowers $(n=5)$ and leaves $(n=1)$ of cannabis plants cultivated by patients enrolled in a safety monitoring project [8] approved by Ethics Committee of Clementino Fraga Filho Hospital, Number 2021817.0.00005257, were donated for this study between 2018 and 2019. Patients received pharmaceutical support from Farmacannabis project to monitor the crops cultivated and preparation of medical cannabis extracts. Cannabis plants were cultivated by clonal propagation, except Medikit CBD strain, which was germinated from a seed. Clonal propagation explants were obtained by cutting and rooting either in rockwool or jiffies at high humidity and light conditions. After root growth, the young plants were transplanted to pots assembled with coconut fiber, perlite, humus and substrate. The plants were maintained under light and moderate humidity at all times until the flowering period begins in indoor (Cinderella, Harle-Tsua and Harle-Tsu ${ }^{b}$ ) and outdoor (Amnesia Haze, Tolomelli and Medikit CBD) cultivation. After completed the vegetative period plants were maintained in a 12-hour light/dark cycle to estimulate the flowering. The flowers were harvested when trichomes showed brown color, except for Harle-Tsu ${ }^{b}$ specimen, which was harvested in the beginning of flowering. The total period between plant rooting and flower harvesting ranged from four to six months. After harvesting, flowers and leaves were separated by grower and maintained in dark and dry conditions. Harle-Tsu ${ }^{\mathrm{a}}$ and Harle-Tsu ${ }^{\mathrm{b}}$ strains were transported to the laboratory still fresh, one day after their harvest. Tolomelli was transferred to lab after ten days and Cinderella, Amnesia Haze and Medikit CBD were taken to lab thirty days after harvest.

In the laboratory, samples were dried in a forced air oven $\left(40{ }^{\circ} \mathrm{C} / 15\right.$ hours $)$ and stored at $-20{ }^{\circ} \mathrm{C}$ until analysis.

\section{Confirmatory method}

Sample preparation

The efficiency between dynamic maceration (DM) and ultrasonic bath (US) as extraction procedures, besides absolute ethanol and methanol:n-hexane 9:1 v/v as extraction solvents, was compared. Dried inflorescences of a hemp strain were grinded and homogenized by mixing. The masses of 50, 100 and 200 mg were extracted by proposed techniques.

The sample to be extracted by DM was transferred to a beaker glass and $10 \mathrm{~mL}$ of extraction solvent was added to be stirred with a magnetic bar for $10 \mathrm{~min}$. The extract was centrifuged at $2007 \mathrm{~g}$ and the supernatant collected. In the extraction by US, samples were transferred to polypropylene tubes and added $10 \mathrm{~mL}$ of extraction solvent, followed by bath in the Elma Schmidbauer ${ }^{\circledR}$ (Singen, Germany) 
Elmasonic E30H during 10 min and followed by centrifugation at $2007 \mathrm{~g}$. In both extraction techniques, the supernatants were collected into $25.00 \mathrm{~mL}$ volumetric flasks and the procedure was repeated twice with the same sample, using 10 and $5 \mathrm{~mL}$ of solvent each time. The extracts were filtered through a $0.22 \mu \mathrm{m}$ membrane and analyzed by HPLC. In order to assess the recovery of extraction in the first-step procedure, the residual sample left after collection of supernatants was fully extracted again two more times and analyzed by HPLC.

\section{HPLC-PDA analysis}

A quantification method by HPLC was developed and validated for five cannabinoids, THCA, THC, CBDA, CBD and CBN. The separation and identification were performed using a Thermo Fisher Scientific ${ }^{\circledR}$ (Breda, The Netherlands) HPLC-PDA equipped with a quaternary pump model 600, type Rheos 5600, an Accela autosampler and a $20 \mathrm{~Hz}$ PDA Accela detector. Data were processed with ChromQuest 5.0 software. The HPLC separation was based on a method previously published [9], after modifications. A C-18 column $(250 \mathrm{~mm} \times 4.6 \mathrm{~mm}$ i.d., $5.0 \mu \mathrm{m})$ purchased from Advanced Chromatography Technologies Ltd. (Aberdeen, UK) was kept at a constant temperature of $30{ }^{\circ} \mathrm{C}$. The mobile phase consisted of (A) 50 $\mathrm{mM}$ ammonium formate buffer, $\mathrm{pH} 5.19$, and (B) methanol at flow rate of $1.0 \mathrm{~mL} \mathrm{~min}^{-1}$. A gradient elution program was conducted as follows: a linear increase of $68 \%-85 \%$ B (0-10 min) and 85\%-92\% B (10-20 min) and finally $95 \%$ B over 5 min. After $25 \mathrm{~min}$, the column was set to the initial condition and re-equilibrated for $5 \mathrm{~min}$. The total processing time was $30 \mathrm{~min}$. Full spectra were recorded in the range $200-400 \mathrm{~nm}$ and quantification was performed at $240 \mathrm{~nm}$. Spectral conditions to calculate the peak purity were wavelength step 1, scan threshold $5 \mathrm{mAU}$ and peak coverage $95 \%$.

\section{Method validation and preparation of secondary reference material}

The method was validated according to the International Council for Harmonization of Technical Requirements for Pharmaceuticals for Human Use (ICH) guideline Q2(R1) [10], the guideline for sanitary regulation of herbal medicines [11] and ANVISA's guideline for analytical method validation, RDC $N^{\circ}$ 166/2017 [12]. The parameters considered for validation were as follows: selectivity, linearity, limit of detection and quantification (LD and LQ, respectively), repeatability (within-day precision) and intermediate repeatability (between-day precision), and accuracy.

The calibration curves were constructed with CRSs. CBDA, CBD, THCA, THC and CBN CRSs were diluted in methanol to obtain a stock solution at concentration $200 \mu \mathrm{g} \mathrm{mL}^{-1}$. The stock solution was diluted to obtain six calibrators whose concentrations were established at 2.00, 5.00, 10.00, 25.00, 50.00 and $110.00 \mu \mathrm{g} \mathrm{mL} \mathrm{m}^{-1}$. Each calibrator was analyzed in triplicate and calibration curves were constructed by linear regression and the coefficient of determination $\left(\mathrm{r}^{2}\right)$ was calculated. The calibration curves were used to quantify the five cannabinoids found in plant samples and to determine their concentration in the secondary reference material (SRM) produced by supercritical fluid extraction (SFE) and used in the repeatability, intermediate repeatability and accuracy assay.

The SRM was prepared from cannabis flowers by supercritical fluid extraction (SFE) in a Top Industrie ${ }^{\circledR}$ (Vaux le Penil, France) $100 \mathrm{~mL}$ model extractor (unpublished data). Dry CBD-rich and THC-rich cannabis flowers previously analyzed by HPLC method were grinded, mixed, inserted into the SFE cell and extracted with $\mathrm{CO}_{2}$ in supercritical state. Methanol was then added to the equipment's separator in order to obtain a methanolic cannabinoid extract. The extract was stored overnight at $-20{ }^{\circ} \mathrm{C}$, filtered through a $0.45 \mu \mathrm{m}$ membrane and quantified according to the calibration standard curves constructed with CBDA, CBD, THCA, THC and CBN CRSs. The SRM was diluted in methanol in order to obtain a solution with approximate concentrations of $2.0 \mathrm{mg} \mathrm{mL}^{-1}$ for THCA and THC, $1.0 \mathrm{mg} \mathrm{mL}^{-1}$ for CBDA and CBD, and 0.5 $\mathrm{mg} \mathrm{mL}^{-1}$ for CBN. The final extract was analyzed by HPLC in eight replicates and the mean concentration of each cannabinoid was measured. The final concentrations were THCA=1.75 $\pm 0.06, \mathrm{THC}=1.64 \pm 0.05$, $\mathrm{CBDA}=1.14 \pm 0.05, \mathrm{CBD}=1.25 \pm 0.05$, and $\mathrm{CBN}=0.53 \pm 0.02 \mathrm{mg} \mathrm{mL}^{-1}$. The SRM was diluted in methanol in order to obtain the quality controls (QCs) used for precision and accuracy assays as shown in Table I. 
Table I. Concentrations of quality controls (QCs) used in precision and accuracy assays

\begin{tabular}{lccc}
\hline \multirow{2}{*}{ Analyte } & \multicolumn{3}{c}{ Concentration $\left[\mu \mathrm{gL}^{-1}\right]$} \\
\cline { 2 - 4 } & $\mathbf{Q C}_{1}$ & $\mathbf{Q C}_{2}$ & $\mathbf{Q C}_{3}$ \\
\hline THCA & 18.0 & 52.0 & 105.0 \\
THC & 16.0 & 49.0 & 98.0 \\
CBDA & 11.0 & 34.0 & 68.0 \\
CBD & 13.0 & 37.0 & 75.0 \\
CBN & 5.0 & 16.0 & 32.0 \\
\hline
\end{tabular}

For precision and accuracy assays, QCs were transferred to polypropylene tubes containing vegetal matrix to mimic the extraction conditions in a real situation. As there is no cannabinoid-free cannabis strain, the cannabis vegetal waste (CVW), which was obtained after cannabinoid extraction by SFE of plants cultivated by patients accompanied by Farmacannabis project for preparation of medicinal extracts, was used as vegetal matrix in precision and accuracy assays. CVWs were collected and analyzed eight times by HPLC to determine the average peak areas for cannabinoids, which were subsequently subtracted from the areas of cannabinoid peaks measured in QCs analysis.

The LD was defined as the lowest concentration of each cannabinoid CRS that yielded a signal greater than noise and a good resolution of peak shape. The lowest concentrations were analyzed in duplicate for three runs and those which reached RSD $\leq 10 \%$ were adopted as LD. The LQ was defined as the lowest point of the calibration curve constructed with CRS in the linear interval from 2.00 to $110.00 \mu \mathrm{g} \mathrm{mL}^{-1}$.

The selectivity was determined by the total peak purity and by analysis of nine non-cannabis species supplied by the Laboratory of Pharmacobotany (Faculty of Pharmacy, Federal University of Rio de Janeiro). The plant species were rhizomes of Glycyrrhiza glabra L. (alcaçuz), dry leaves of Artemisia annua, Atropa belladonna L., Brugmansia suaveolens, Erythroxylum coca, Digitalis purpurea, Mentha spicata, seeds of Paullinia cupana (guarana) and fruits of Cola nitida (cola nut). The non-cannabis species were selected based on availability of botanically characterized species. Considering that illicit cannabis can be diluted with several non-cannabis specimens, species containing psychoactive compounds, such as alkaloids and xanthenes, were also included.

\section{Presumptive method}

Beam test is based on an alkaline solution that reacts with CBD, resulting in a purple violet color $[13,14]$. The Beam reagent was prepared by $98 \%$ dilution of sodium hydroxide $(\mathrm{NaOH})$ in absolute ethanol or in a hydroalcoholic solution (absolute ethanol:ultrapurified water, 1:1 v/v) to obtain three solutions as follows: (1) $1 \mathrm{~mol} \mathrm{~L}^{-1} \mathrm{NaOH}$ alcoholic solution; (2) $1 \mathrm{~mol} \mathrm{~L}^{-1} \mathrm{NaOH}$ hydroalcoholic solution and (3) $2.5 \mathrm{~mol} \mathrm{~L}^{-1} \mathrm{NaOH}$ hydroalcoholic solution.

Dry flowers $(10 \mathrm{mg})$ from same cannabis samples previously analyzed by HPLC were grinded and transferred to the center of a circular filter paper weight $80 \mathrm{~g} \mathrm{~m}^{-2}, 70 \mathrm{~mm}$ diameter. Beam modified reagent was dripped on the sample with a Pasteur pipette, 3 drops in the center of sample. The color was observed after 5 minutes. 


\section{Data analysis}

Microsoft excel and GraphPad Prism 8.4.3 software were used for table plot and statistical analysis, respectively. The data of extraction optimization were compared by the Kruskal-Wallis test, followed by the Dunn's post hoc test at the $0.5 \%$ level of significance.

\section{RESULTS AND DISCUSSION HPLC method}

HPLC is the best choice in situations where it is required to differentiate cannabinoids in acid and neutral forms because the analysis does not require heating, in contrast to gas chromatography methods that favors decarboxylation $[9,15]$. The quality of raw medical cannabis material needs to be determined in order to differentiate acid and neutral forms and to clarify the conditions of cannabis storage that can be also useful for police intelligence. For instance, higher concentrations of acid forms found in fresh flowers can indicate recent harvesting or mild storage temperatures.

The cannabinoid extraction technique provides clean chromatograms with minimal interferences. Extraction with organic solvents is simple compared to other techniques, such as solid-phase extraction. Besides, methanol:chloroform 9:1 v/v, methanol:n-hexane 9:1 v/v and ethanol-based extractions are often described in HPLC methods [6,9,16-18]. Extraction with methanol:n-hexane 9:1 v/v mixture over a period of 10 to 30 minutes shows good cannabinoid yields in a single step [6].

In the extraction optimization assays, we compared the efficiency of extraction using different sample weights. The concentration of CBD extracted from dried plants was affected by the amount of sample analyzed $(\mathrm{H}=7.200$, Df $=2, \mathrm{p}$-value $=0.003)$, with $50 \mathrm{mg}$ samples showing a slightly increased CBD concentration than $100 \mathrm{mg}$ samples ( $p$-value $=0.021$ ). CBDA and THCA extractions were not affected by initial weight of plant material $(H=5.067, \mathrm{Df}=2$, $p$-value $=0.085$ for $\mathrm{CBDA}$; and $\mathrm{H}=5.422$, $\mathrm{Df}=2$, $p$-value $=0.071$ for THCA; Figure 1.A). Furthermore, masses of 50, 100 and $200 \mathrm{mg}$ showed a CBDA yield of more than $99 \%$ in the first extraction and CBDA concentrations near the detection limit for the second and third extraction (Figure 1.B). Next, we evaluated the impact of different extraction techniques on the total concentration of cannabinoids. Even though there was a significant difference between medians obtained for CBD and THCA (H = 9.359, Df = 2, p-value = 0.002 for CBD; and H=7.423, Df = 2, $\mathrm{p}$-value $=0.029$ for THCA; Figure 2), follow-up analysis by Dunn's post hoc test did not show differences that reached the significance threshold established in our protocol $(\alpha=5 \%)$ for any groups. Such difference was not identified for $\mathrm{CBDA}(\mathrm{H}=6.897, \mathrm{Df}=2$, $\mathrm{p}$-value = 0.050; Figure 2). It was selected the extraction technique based on $100 \mathrm{mg}$ of vegetal mass in methanol:n-hexane 9:1 v/v because this same solvent is used to extract cannabis oily medicinal extracts in our laboratory [19] and $100 \mathrm{mg}$ is the mass used to analyze terpenes by a non-destructive GC-MS method [20]. 

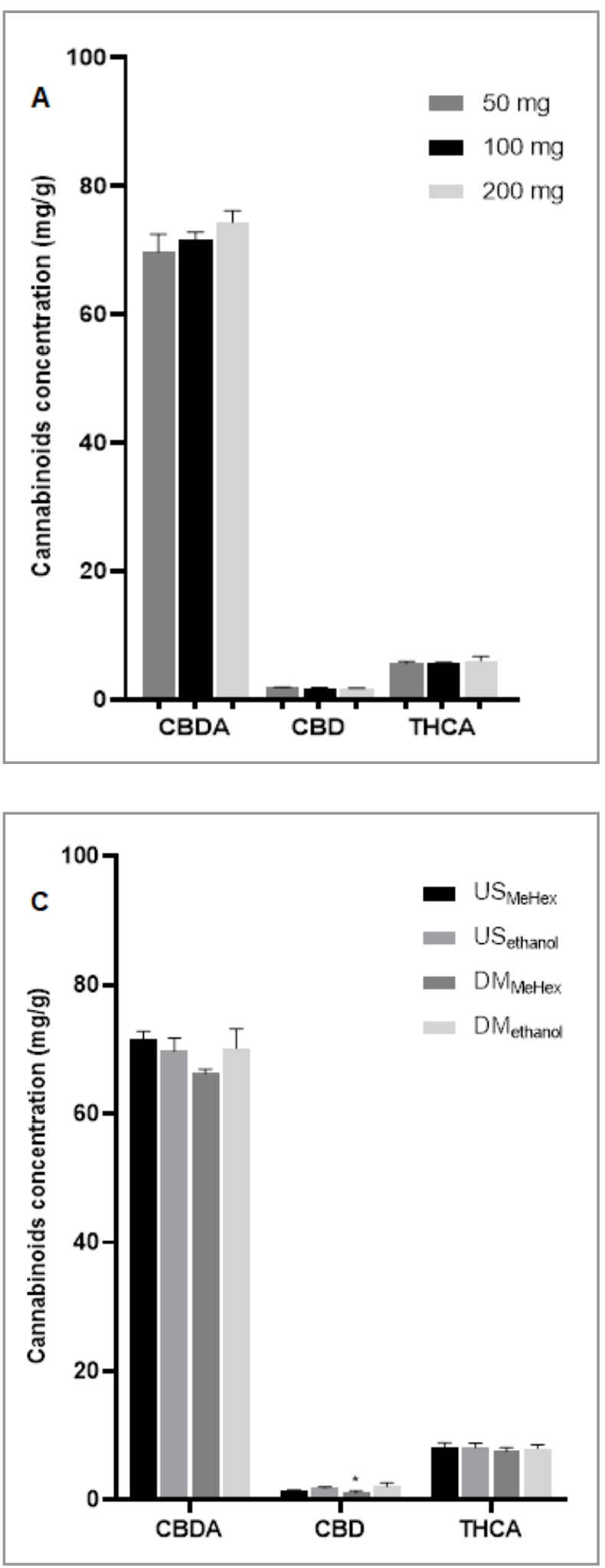

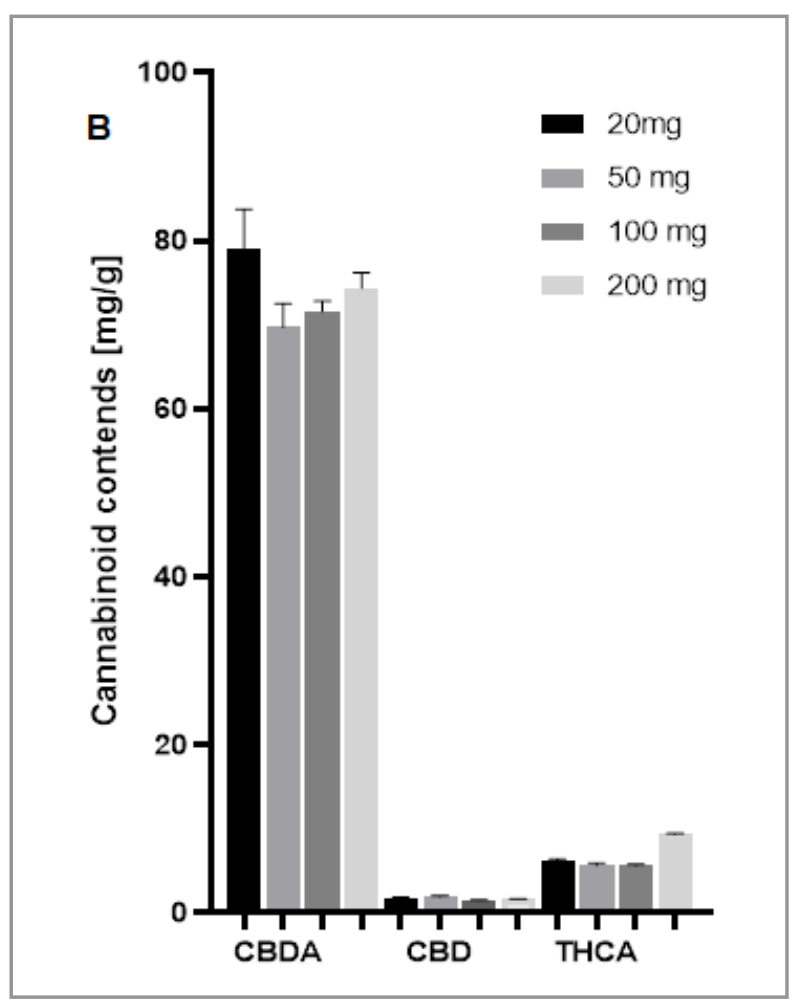

Figure 1. Comparison of cannabinoid extraction according to sample mass and extraction technique. A: Sample mass data analyzed by the Kruskal-Wallis test followed by Dunn's post hoc test; B: CBDA yields in the first, second and third extraction (CBD and THCA were not detected from the second extraction and therefore no data are shown for these compounds); C: Extraction technique data were analyzed by the KruskalWallistest, followed by Dunn's post hoc test. *p-value $<0.05$. Note: US $_{\text {MeHex }}$ : ultrasound with methanol:n-hexane $(9: 1 \mathrm{v} / \mathrm{v})$; US $_{\text {ethano: }}$ : ultrasound with ethanol; $\mathrm{DM}_{\text {MeHex: }}:$ dynamic maceration with methanol:n-hexane $(9: 1 \quad \mathrm{v} / \mathrm{v}) ; \quad \mathrm{DM}_{\text {ethanol: }}$ dynamic maceration with ethanol.

Typical HPLC cannabis chromatograms (Figures 2 and 3) differed from those of other plant species such as Atropa belladonna (Figure 4), even in comparison with the CVW (Figure 5). The chromatographic conditions were selective in the separation of five cannabinoids, the peaks showed good shape and good resolution (Figure 6), with minimal interferences. 
Plant analysis is a forensic challenge because the chemical profile varies according to genotype and cultivation conditions (temperature, soil quality, humidity, light conditions, etc.) and a blank is not available since a plant is not expected to be free of typical secondary metabolites [21]. However, the chromatographic profiles of authentic samples were typical and can be combined with the spectra of cannabinoid reference standards to confirm the Cannabis genus.

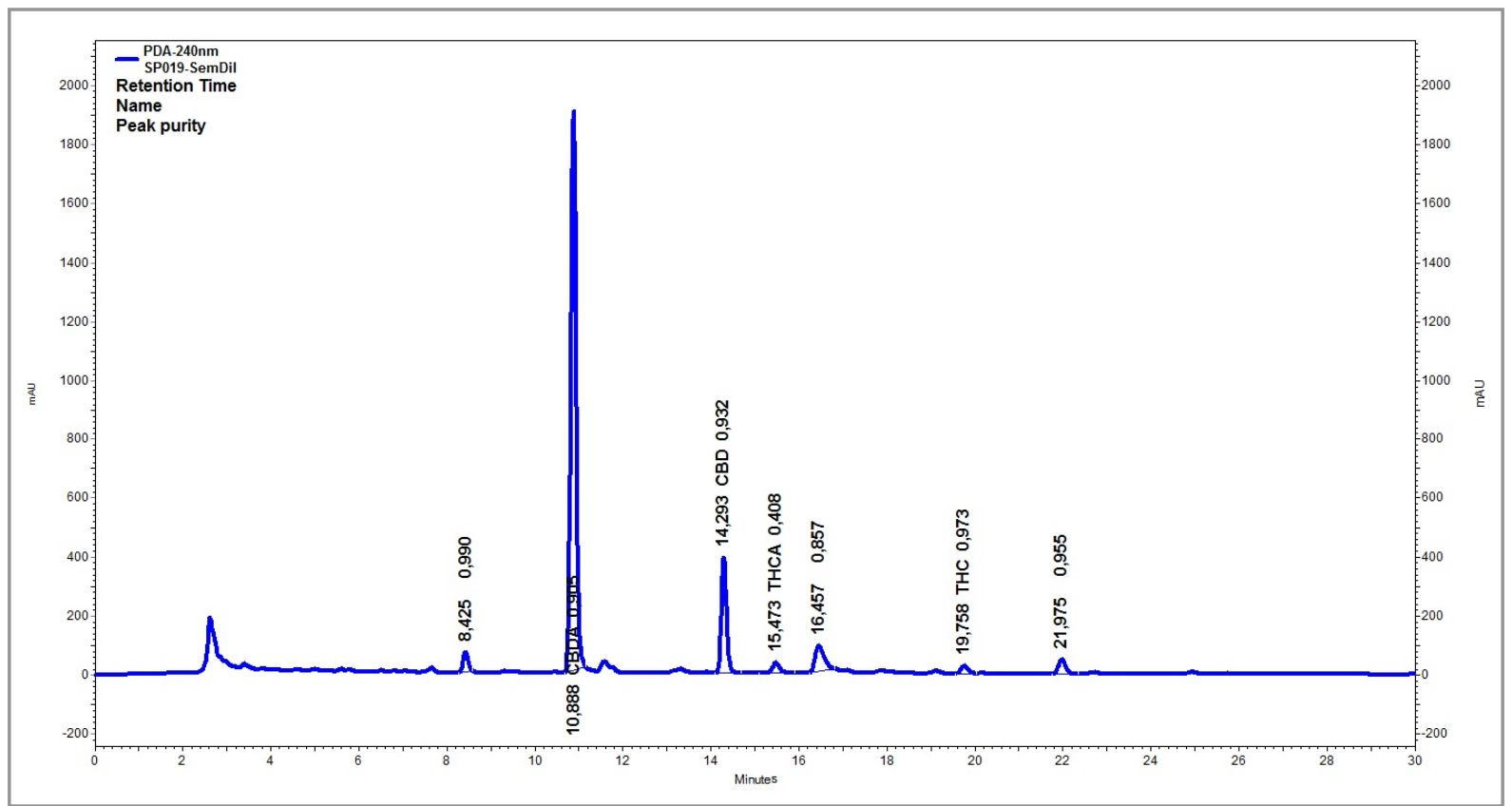

Figure 2. Chromatographic profile of the Harle-Tsu strain of cannabis.

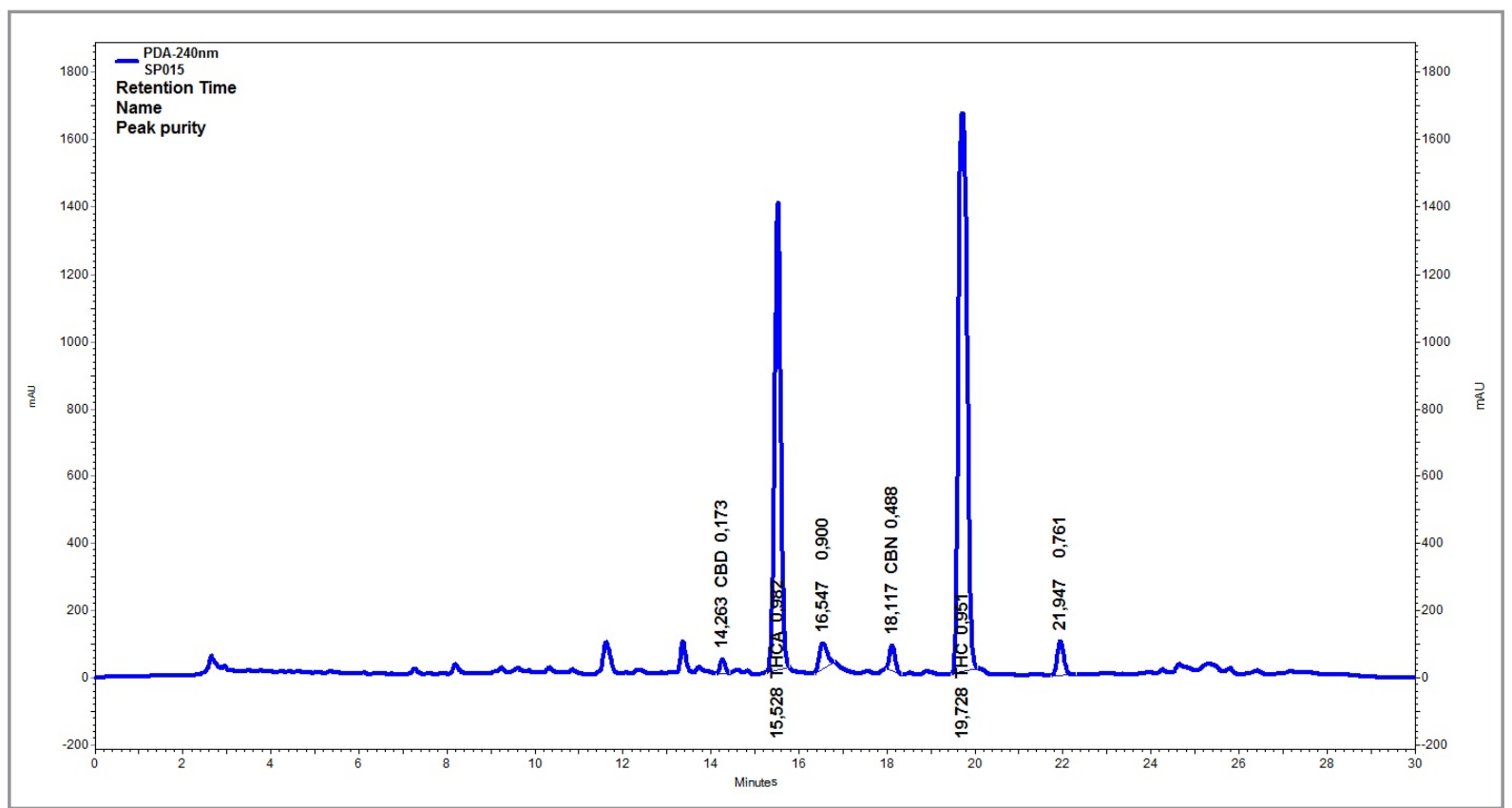

Figure 3. Chromatographic profile of the Amnesia Haze strain of cannabis. 


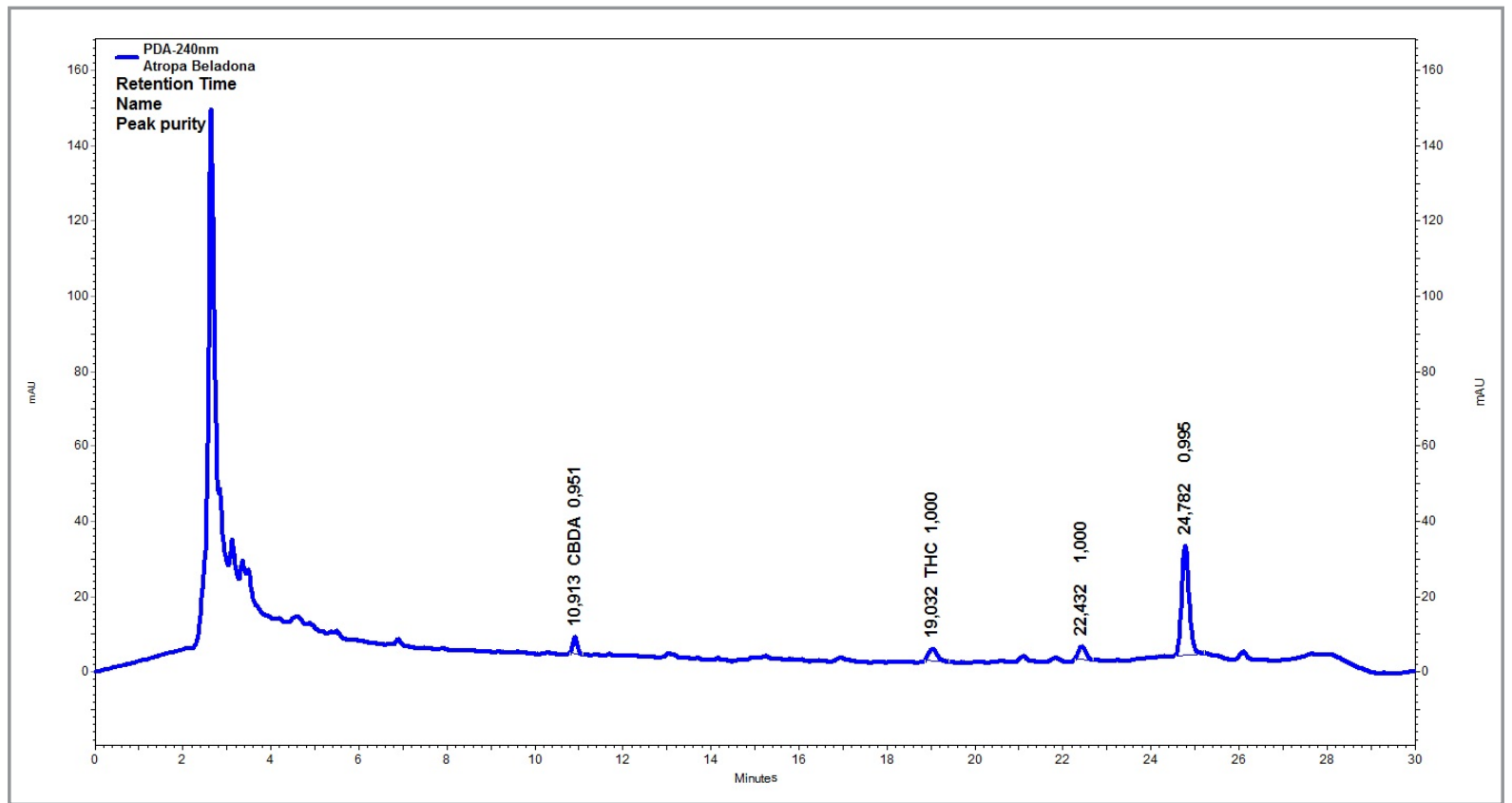

Figure 4. Chromatographic profile of the Atropa belladonna leaves.

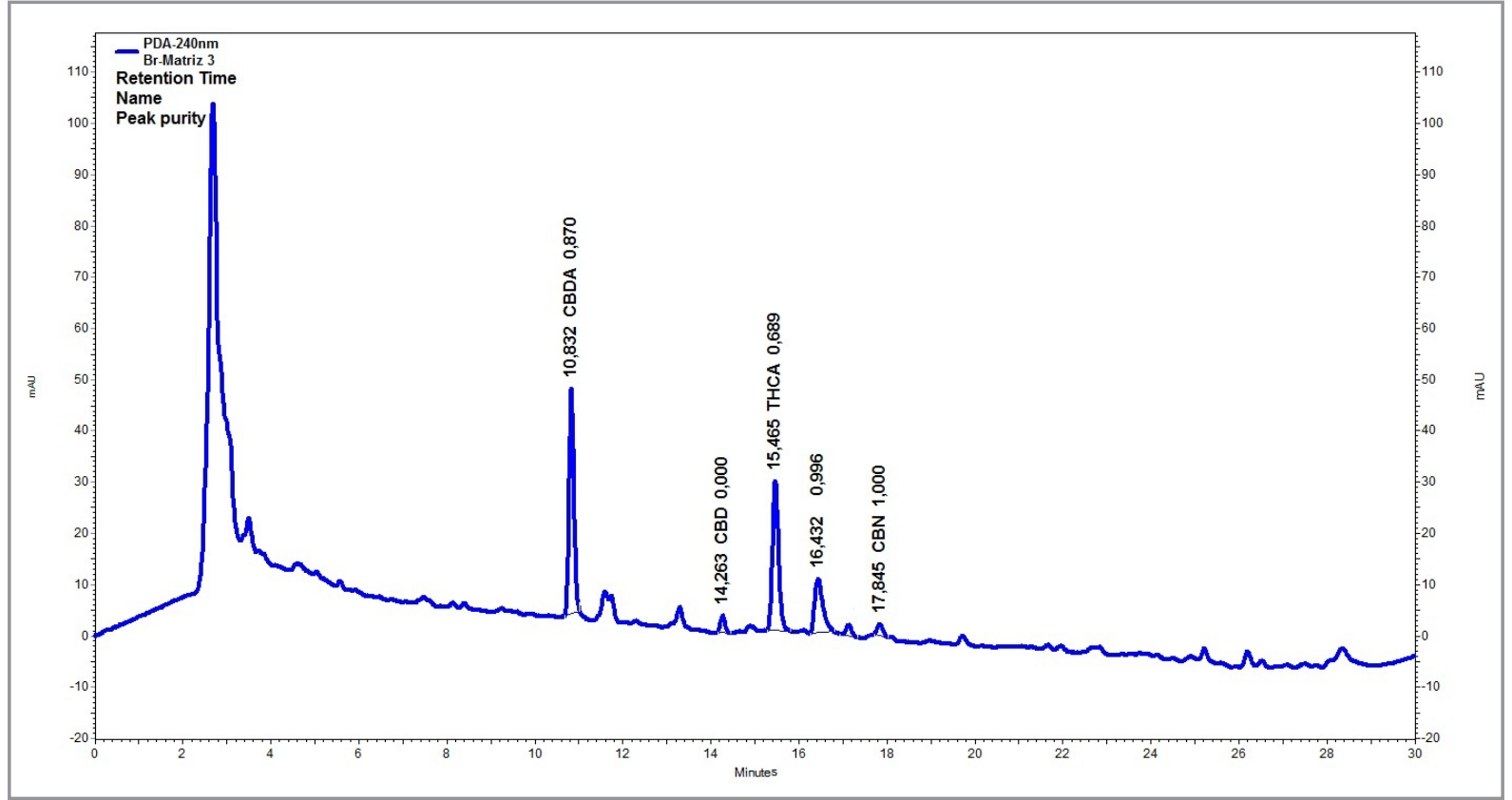

Figure 5. Chromatographic profile of the cannabis vegetable waste after extraction by SFE. 


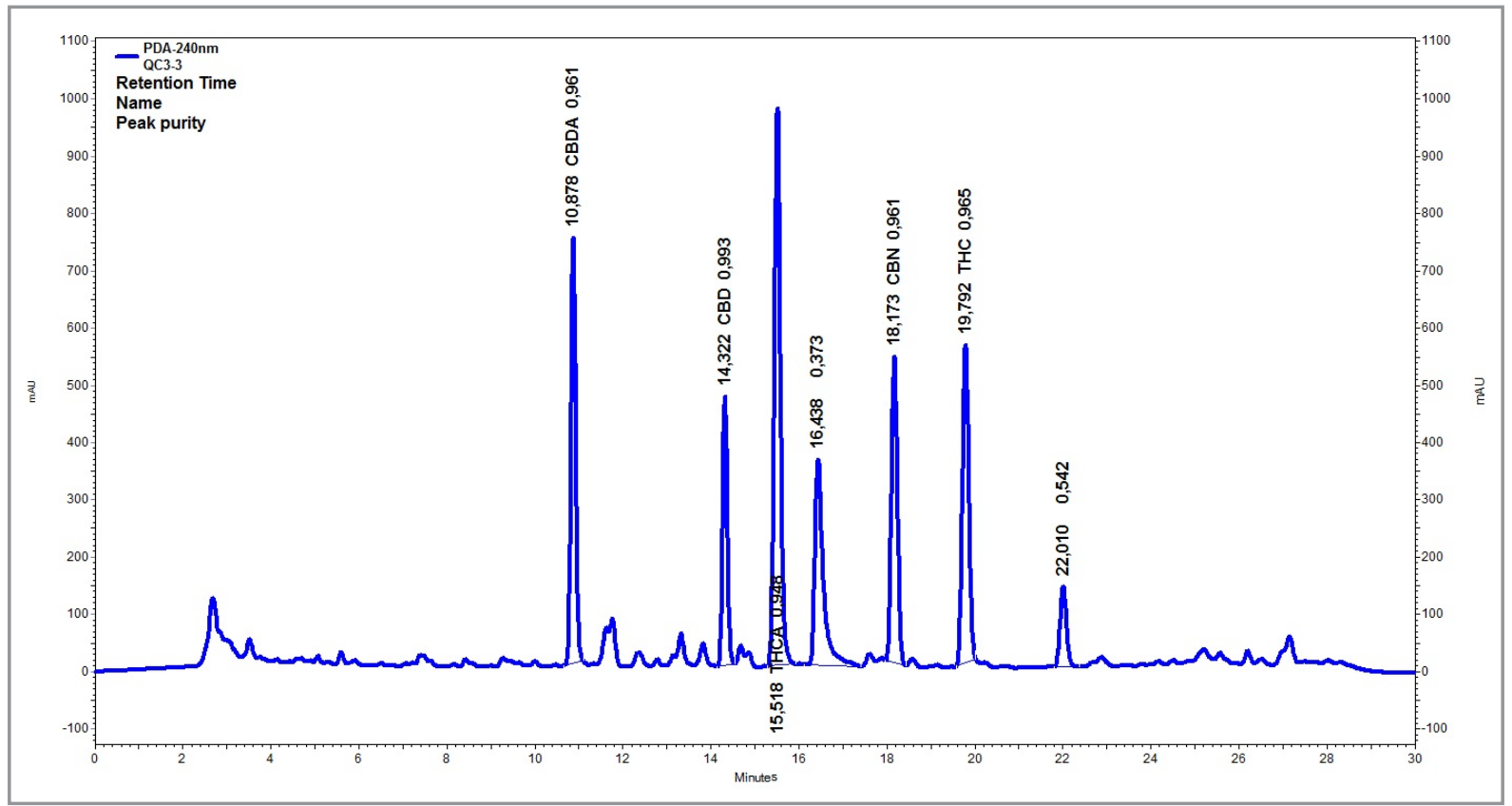

Figure 6. Chromatographic profile of QC3 solution.

Note: Cannabinoid concentrations: $\mathrm{CBDA}=68.0, \mathrm{CBD}=75.0, \mathrm{THCA}=105.0, \mathrm{CBN}=32.0, \mathrm{THC}=98.0 \mu \mathrm{gL}^{-1}$.

Unlike most countries in North America and Europe, the purchase of cannabinoid CRS in Brazil is an obstacle for method validation. There are no national suppliers and importation has become more difficult with the COVID-19 pandemic. Due to the lack of reference standards from plant-derived drugs, Brazilian regulation accepts secondary reference standards (SRS) to validate analytical methods applied to herbal medicines [11,22]. Thus, a methanolic cannabinoid extract prepared by SFE was used as SRS.

A blank plant matrix is not often available because secondary metabolites will always be present in plants. Thus, a cannabis vegetable waste after extraction by SFE (Figure 5) was used as reference matrix for precision and accuracy assay by a spiked technique. The validation results (Table II) satisfied the criteria recommended for herbal medicines [11,22]. LD and LQ were below the cannabinoid levels previously quantified in cannabis leaves (between $1.10 \%$ and $2.10 \%$ ) and inflorescences (between $15.77 \%$ and $20.37 \%$ ) [6].

Table II. Validation results obtained by HPLC for the quantification of cannabinoids in cannabis samples

\begin{tabular}{llllll}
\hline Parameter & THCA & THC & CBDA & CBD & CBN \\
\hline LD $\left(\mu \mathrm{g} \mathrm{mL}^{-1}\right)$ & 0.40 & 0.70 & 0.60 & 0.80 & 0.50 \\
LD $\left(\mathrm{mg} \mathrm{g}^{-1}\right)$ & 0.10 & 0.18 & 0.15 & 0.20 & 0.12 \\
LQ $\left(\mu \mathrm{g} \mathrm{mL}^{-1}\right)$ & 2.00 & 2.00 & 2.00 & 2.00 & 2.00 \\
LQ $\left(\mathrm{mg} \mathrm{g}^{-1}\right)$ & 0.50 & 0.50 & 0.50 & 0.50 & 0.50 \\
Linearity & 0.999898 & 0.999995 & 0.999708 & 0.999991 & 0.999992 \\
2.00 to $110 \mu \mathrm{g} \mathrm{mL}^{-1}\left(\mathrm{r}^{2}\right)$ & & & &
\end{tabular}


Carvalho, V. M.; de Almeida, F. G.; de Aguiar, A. F. L.; Pereira, F. C. S.; Rocha, E. D.

Table II. Validation results obtained by HPLC for the quantification of cannabinoids in cannabis samples (Cont)

\begin{tabular}{lccccc} 
Parameter & THCA & THC & CBDA & CBD & CBN \\
\hline Repeatability (\%RSD) & & & & & \\
\hline QC1 & 3.18 & 3.33 & 1.69 & 3.20 & 1.93 \\
QC2 & 0.32 & 1.44 & 0.38 & 0.40 & 0.31 \\
QC3 & 1.60 & 1.58 & 1.42 & 1.46 & 1.51 \\
\hline Intermediate repeatability (\%RSD) & & & & \\
\hline QC1 & 4.27 & 3.86 & 3.33 & 2.66 & 3.82 \\
QC2 & 1.47 & 2.02 & 2.98 & 2.23 & 1.79 \\
QC3 & 1.83 & 1.99 & 2.16 & 1.16 & 2.14 \\
\hline Recovery (\%RSD) - Accuracy & & & & & \\
\hline QC1 & 76.92 & 109.76 & 88.32 & 94.77 & 114.82 \\
QC2 & 82.07 & 119.28 & 94.71 & 99.45 & 116.85 \\
QC3 & 79.95 & 115.06 & 91.52 & 96.07 & 113.21 \\
\hline
\end{tabular}

$L D=$ limit of detection; $L Q=$ limit of quantification; $L D$ and $L Q$ were determined for cannabinoid $C R S s\left(\mu \mathrm{mL}^{-1}\right)$ and estimated for vegetal samples ( $\left.\mathrm{mg} \mathrm{g}^{-1}\right)$; $\mathrm{QC}=$ quality control in $\mu \mathrm{g} \mathrm{mL}^{-1}$ is shown in Table I; RSD=relative standard deviation.

The proposed method was applied to five samples of cannabis inflorescences and one sample of cannabis leaves and tree different profiles were obtained: CBD-rich, THC-rich and approximately 1:1 THC:CBD ratio (Table III). Total CBD and total THC values were calculated by the acid form plus the neutral form multiplied by the factor 0.88 , which was used to adjust for the different molecular weights of the cannabinoid and carboxylic conjugative components: 314.46/358.47 for THC/THCA and 314.46/358.47 for CBD/CBDA [23].

Concentrations of neutral and acid THC and CBD forms are used to identify marijuana and hemp plants types $[6,24]$. In this study, five cannabinoids (CBDA, CBD, THCA, THC and CBN) were present in most samples, except for sample with immature flowers (Harle-Tsu ${ }^{b}$ ) containing 1.6\% CBD and THC-free.

Table III. Cannabinoid content in six authentic samples analyzed by HPLC method

\begin{tabular}{lccccccc|cc|c|c|c}
\hline & \multicolumn{7}{c|}{ Concentration [mg g-1 } & & $\%$ & Ratio & Classification \\
\hline Variety & CBDA & CBD & THCA & THC & CBN & CBD $_{\text {total }}$ & THC $_{\text {total }}$ & CBD & THC & THC/CBD & \\
\hline Cinderella & 4.23 & 1.06 & 12.76 & 0.11 & 0.33 & 4.78 & 11.34 & 0.48 & 1.13 & 0.42 & marijuana \\
Amnesia Haze & 3.28 & 1.80 & 33.57 & 170.24 & 1.70 & 4.68 & 199.78 & 0.47 & 19.98 & 0.02 & marijuana \\
Tolomelli & 37.83 & 4.52 & 26.71 & 12.29 & 1.06 & 37.81 & 35.79 & 3.78 & 3.58 & 1.06 & intermediate \\
Harle-Tsu & 60.04 & 6.30 & 6.29 & 1.91 & nd & 59.14 & 7.45 & 5.91 & 0.74 & 7.94 & hemp \\
Harle-Tsu & 15.55 & 2.51 & nd & nd & nd & 16.19 & 0,00 & 1.62 & 0.00 & THC free & hemp \\
Medikit CBD & 75.88 & 100.31 & 4.84 & 5.67 & 1.55 & 167.08 & 9.93 & 16.71 & 0.99 & 16.83 & hemp \\
\hline
\end{tabular}

Note: $\mathrm{CBD}_{\text {total }}=\mathrm{CBDA}^{*} 0.88+\mathrm{CBD} ; \mathrm{THC}_{\text {total }}=\mathrm{THCA}^{*} 0.88+\mathrm{THC}=; 0.88$ factor: $\mathrm{CO}_{2}$ lost in the decarboxylation; nd: not detected. 


\section{Presumptive color test}

Six cannabis samples analyzed by HPLC (Table III) were used to standardize the presumptive color test proposed to differentiate CBD-rich and THC-rich cannabis strains.

The Beam reagent was referred as a specific color test for CBD with positive result characterized by purple violet color $[13,14]$. The presumptive test was developed with a $\mathrm{NaOH}$ solution dripped on a $10 \mathrm{mg}$ of pulverized dry sample, placed in the center of a circular filter paper. The color test has shown positive results in a presumed CBD concentration approximately above $2 \%$ (Figure 7 ). CBD-rich samples showed purple violet color based on CBD content. Considering the intensity of color reaction, the purple violet color was more intense for Medikit CBD, Harle-Tsu and Harle-Tsu ${ }^{b}$, suggesting a possible correlation between CBD concentrations and color intensity. THC-rich samples, such as Cinderella and Amnesia Haze strains, showed no color change and samples with CBD and THC contents of approximately 1:1 w/w were positive (purple color). The Beam reagent was proposed in the last century as a color test reagent for the identification of cannabis samples. It was described as a $5 \%$ of potassium hydroxide solution in ethanol [25] and a $2 \%$ potassium hydroxide solution in ethanol or acidic medium (95\% ethanol saturated with $\mathrm{HCl}$ ) [14], but its use is no longer accepted by forensic laboratories because seized cannabis material has failed to yield a positive reaction with either reagent. The test was later shown to be specific for CBD, while no reaction is led by $\mathrm{THC}$ content [14]. In our study, acid solutions $\left(\mathrm{HCl} 1 \mathrm{~mol} \mathrm{~L}^{-1}\right.$ alcoholic and $\mathrm{HCl}$ $1 \mathrm{~mol} \mathrm{~L}^{-1}$ hydroalcoholic solutions) were tested on the six cannabis samples and the results were negative in all assays (no color change).

Nowadays it is important to identify CBD-rich and THC-rich cannabis species for the medical and forensic areas. In the present study, a reagent consisting of a 1 to $2.5 \mathrm{~mol} \mathrm{~L}^{-1} \mathrm{NaOH}$ alcoholic or hydroalcoholic solutions was able to identify CBD levels above $16 \mathrm{mg} \mathrm{g}^{-1}$ in dry samples. THC-rich samples with CBD levels below $5 \mathrm{mg} \mathrm{g}^{-1}$ showed negative results characterized by no color change.

The $1 \mathrm{~mol} \mathrm{~L}^{-1} \mathrm{NaOH}$ alcoholic solution showed more intense colorations and provided the best visualization of colors, especially for Harle-Tsu ${ }^{b}$ and a CBD level $16 \mathrm{mg} \mathrm{g}^{-1}$ which is suggested to be the LD.

Many patients enrolled in the Farmacannabis project reported buying Beam reagent on the internet, where it is sold as a rapid simple test for the identification of CBD in plants and cannabis extracts. However, the test does not identify THC-rich plants, besides THC/CBD ratio of approximately 1:1 w/w also result in purple violet color. The test is not useful to discriminate medical cannabis strains when THC represents a risk for treatment. For instance, epileptic children under treatment with CBD-rich cannabis may suffer increased seizure frequency if their extracts are prepared with a cannabis strain containing a THC:CBD ratio of approximately $1: 1 \mathrm{w} / \mathrm{w}$.

The color test proved to be useful for forensic laboratories as a presumptive rapid test to discriminate hemp and marijuana cannabis strains and can be combined with botanic analysis and other classical color tests (Fast Blue B and Duquenois-Levine) to identify high-THC cannabis (marijuana or drug-type). For instance, a negative result in modified Beam color test and positive result in Fast Blue or Duquenois combined with a positive result in botanic analysis suggest a marijuana strain. 

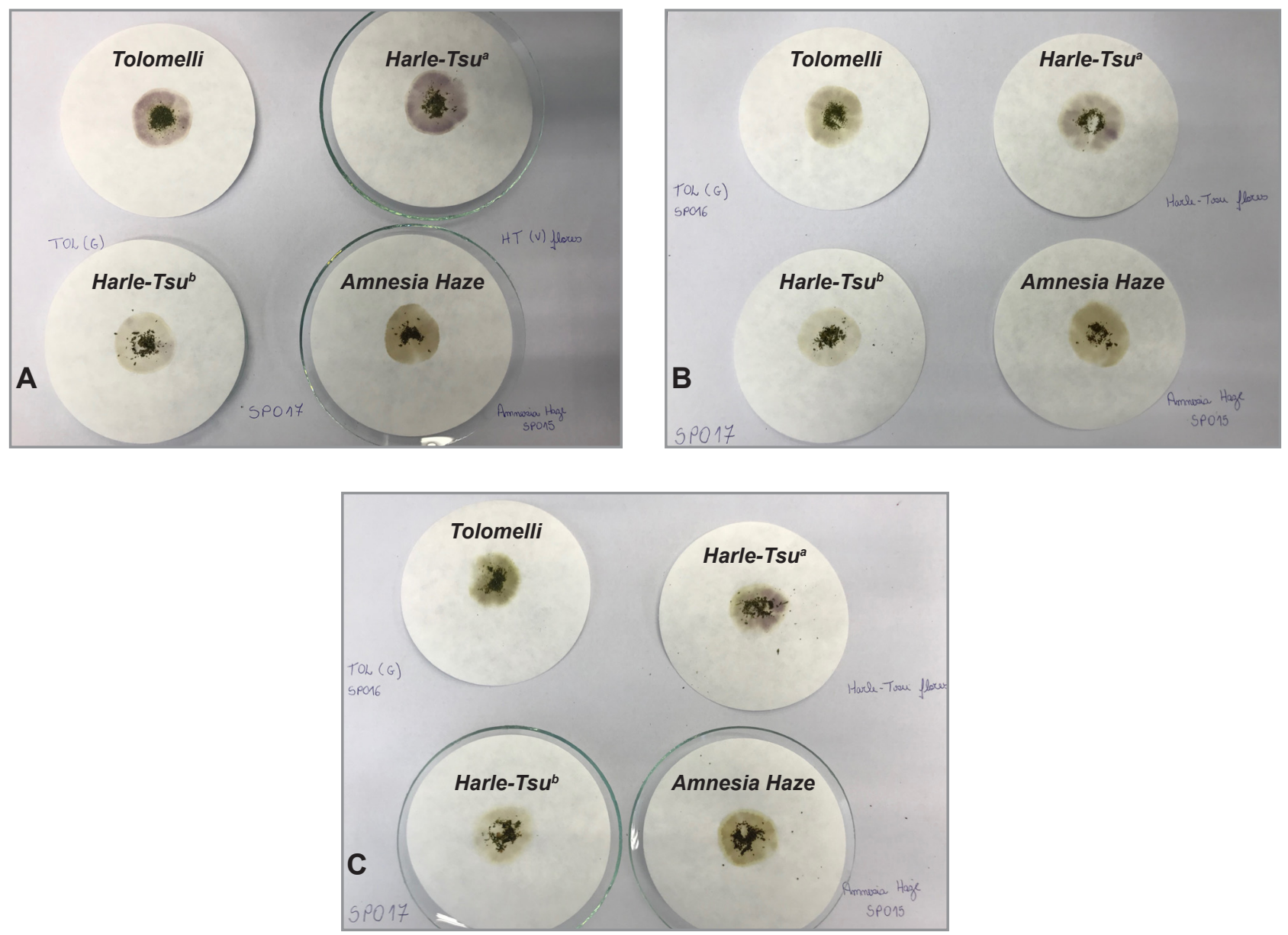

Figure 7. Modified Beam color test applied to cannabis strains. [A] $\mathrm{NaOH}$ alcoholic $1 \mathrm{~mol} \mathrm{~L}^{-1}$; [B] NaOH hydroalcoholic $1 \mathrm{~mol} \mathrm{~L}^{-1} ;$ [C] NaOH hydroalcoholic $2.5 \mathrm{~mol} \mathrm{~L}^{-1}$.
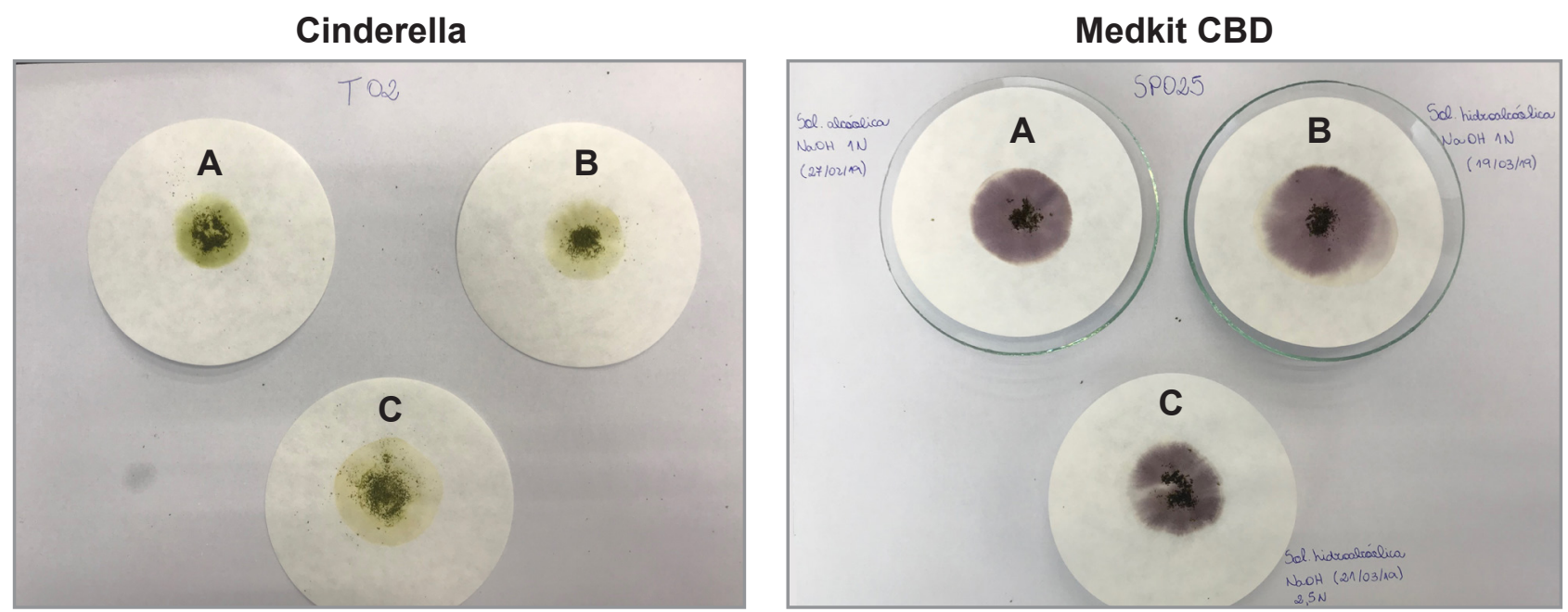

Figure 8. Modified Beam color test applied to cannabis strains. [A] NaOH alcoholic solution $1 \mathrm{~mol} \mathrm{~L}^{-1}$; [B] NaOH hydroalcoholic solution $1 \mathrm{~mol} \mathrm{~L}^{-1} ;$ [C] NaOH hydroalcoholic solution $2.5 \mathrm{~mol} \mathrm{~L}^{-1}$. 


\section{CONCLUSIONS}

The proposed methodology, consisting of presumptive and confirmatory methods, is simple and less expensive compared to mass spectrometry-based methods, which could lead to its implementation in most Brazilian laboratories either for forensic purposes or sanitary inspections.

The proposed presumptive color test detects CBD at concentrations above $16 \mathrm{mg} \mathrm{g}^{-1}$ in minimal sample quantities (10 $\mathrm{mg}$ ) and is useful as a presumptive test to differentiate marijuana from hemp-types, but not from intermediate types (THC:CBD ratio of approximately $1: 1 \mathrm{w} / \mathrm{w}$ ).

The cannabinoid profile of the cannabis samples donated by patients indicates three cannabis types, i.e., marijuana, hemp and intermediate types. The medical cannabis profile used in Brazil should be better investigated after increasing the sample size.

\section{Conflicts of interest}

We wish confirm that there are no known conflicts of interest associated with this publication and there has been no significant financial support for this work that could have influenced its outcome.

\section{Acknowledgements}

The authors gratefully acknowledge financial support from the Serrapilheira Institute [grant number Serra-1709-18891], "Conselho Nacional de Desenvolvimento Científico e Tecnológico" (CNPQ), Brazil [Process Number-426406/2018-4], from the "Fundação Carlos Chagas Filho de Amparo à Pesquisa do Estado do Rio de Janeiro" [Process Numbers E26 010.000230/2017 and E26/210.260/2018] and scholarship awarded for Fernanda Cardosos dos Santos Pereira [Process Number E-26/202.977/2018].

The authors gratefully acknowledge technical support from the Prof. Dr. Antônio Jorge Ribeiro da Silva, "Instituto de Pesquisas de Produtos Naturais Walter Mors", UFRJ, for use of supercritical fluid extraction equipment.

\section{REFERENCES}

1. Mead, A. Epilepsy Behav., 2017, 70, pp 288-291 (https://doi.org/10.1016/j.yebeh.2016.11.021).

2. Cox, C. Health Policy, 2021,125 (1), pp 12-16 (https://doi.org/10.1016/j.healthpol.2020.10.016).

3. United Nations Office on Drugs and Crime. Recommended methods for the identification and analysis of cannabis and cannabis products. New York, 2012.

4. Hillig, K. W.; Mahlberg, P. G. Am. J. Bot., 2004, 91 (6), pp 966-975 (https://doi.org/10.3732/ajb.91.6.966).

5. Sawler, J.; Stout, J. M.; Gardner, K. M.; Hudson, D.; Vidmar, J.; Butler, L.; Page, J. E.; Myles, S. PLoS One, 2015, 10 (8), e0133292 (https://doi.org/10.1371/journal.pone.0133292).

6. Jin, D.; Dai, K.; Xie, Z.; Chen, J. Sci. Rep., 2020, 10 (1), pp 1-14 (https://doi.org/10.1038/s41598-02060172-6).

7. Romero, P.; Peris, A.; Vergara, K.; Matus, J. T. Plant. Sci., 2020, 298, 110571 (https://doi.org/10.1016/j. plantsci.2020.110571).

8. Carvalho, V. M. Braz. J. Anal. Chem., 2017, 4 (16), pp 44-49.

9. De Backer, B.; Debrus, B.; Lebrun, P.; Theunis, L.; Dubois, N.; Decock, L.; Verstraete, A.; Hubert, P.; Charlier, C. J. Chromatogr. B, 2009, 877 (32), pp 4115-4124 (https://doi.org/10.1016/j. jchromb.2009.11.004).

10. International Council for Harmonisation of Technical Requirements for Pharmaceuticals for Human Use. ICH Q2(R1) guideline. Validation of analytical procedures: Text and Methodology, 2005.

11. Agência Nacional de Vigilância Sanitária, ANVISA. Instrução Normativa $n^{\circ} 4$ - Guia de orientação para registro de Medicamento Fitoterápico e registro e notificação de Produto Tradicional Fitoterápico, Brasília, 2014.

12. Agência Nacional de Vigilância Sanitária, ANVISA. Resolução da Diretoria Colegiada - RDC $n^{\circ} 166$, Brasília, 2017. 
13. Thornton, J. I.; Nakamura, G. R. J. Forensic. Sci. Soc., 1972, 12 (3), pp 461-519 (https://doi.org/10.1016/ s0015-7368(72)70716-1).

14. Spiro, B. US4771005A, September 13, 1988, Erez Forensic Technology Ltd., Israel.

15. Patel, B.; Wene, D.; Fan, Z. T. J. Pharm. Biomed. Anal., 2017, 146, pp 15-23 (https://doi.org/10.1016/j. jpba.2017.07.021).

16. Ambach, L.; Penitschka, F.; Broillet, A.; König, S.; Weinmann, W.; Bernhard, W. Forensic Sci. Int., 2014, 243, pp 107-111 (https://doi.org/10.1016/j.forsciint.2014.06.008).

17. Gul, W.; Gul, S. W.; Radwan, M. M.; Wanas, A. S.; Mehmedic, Z.; Khan, I. I.; Sharaf, M. H.; ElSohly, M. A. J. AOAC Int., 2015, 98 (6), pp 1523-1528 (https://doi.org/10.5740/jaoacint.15-095).

18. Berman, P.; Futoran, K.; Lewitus, G. M.; Mukha, D.; Benami, M.; Shlomi, T.; Meiri, D. Sci. Rep., 2018, 8 (1), pp 1-15 (https://doi.org/10.1038/s41598-018-32651-4).

19. Carvalho, V. M.; Aguiar, A. F.; Baratto, L. C.; Souza, F. L.; Rocha, E. D. Quim. Nova, 2020, 43 (1), pp 90-97 (https://doi.org/10.21577/0100-4042.20170457).

20. Rocha, E. D.; Silva, V. E.; Pereira, F.; Jean, V. M.; Souza, F. L. C.; Baratto, L. C.; Vieira, A.; Carvalho, V. M. Rodriguésia, 2020, 71, e01192019 (https://doi.org/10.1590/2175-7860202071040).

21. Vickery, C. R.; La Clair, J. J.; Burkart, M. D.; Noel, J. P. Curr. Opin. Chem. Biol., 2016, 31, pp 66-73.

22. Swift, W.; Wong, A.; Li, K. M.; Arnold, J. C.; McGregor, I. S. PLoS One, 2013, 8 (7), e70052 (https://doi. org/10.1371/journal.pone.0070052).

23. Richins, R. D.; Rodriguez-Uribe, L.; Lowe, K.; Ferral, R.; O'Connell, M. A. PLoS One, 2018, 13 (7), e0201119 (https://doi.org/10.1371/journal.pone.0201119).

24. Lau-Cam, J.; McDonnell, C. A. Bull. Narcotics, 1978, 30, pp 63-68. Available at: https://www.unodc. org/unodc/en/data-and-analysis/bulletin/bulletin_1978-01-01_2_page008.html [Accessed 02 March 2021]. 